Pacific Journal of Mathematic 


\section{A RADON NIKODYM THEOREM FOR WEIGHTS ON VON NEUMANN ALGEBRAS}

\section{A. VAN DEALE}

Let $\varphi$ and $\psi$ be normal positive linear functionals on a von Neumann algebra $M$ such that $\psi \leqq \varphi$. Sakai proved the existence of a unique element $h \in M$ with $0 \leqq h \leqq 1$ such that $\psi(x)=\frac{1}{2} \varphi(h x+x h)$ for any $x \in M$. A generalization of this theorem is obtained for weights on von Neumann algebras. Let $\varphi$ be a faithful normal semi-finite weight and $\psi$ any weight on $M$ majorized by $\varphi$. Then there is a unique element $h \in M$ with $0 \leqq h \leqq 1$ such that $\psi(x)=\frac{1}{2} \varphi(h x+x h)$ holds for $x$ in a $\sigma$-weakly dense *-subalgebra of $M$. A stronger version is obtained when $\psi$ is assumed to be a normal positive linear functional. Moreover counterexamples are given to show that in general one can not expect this relation to hold for every $x \in M^{+}$.

1. Introduction. Let $M$ be a von Neumann algebra with a faithful normal state $\varphi$. Sakai proved that for any positive linear functional $\psi$ on $M$ such that $\psi \leqq \varphi$ there exists a unique element $h \in M$ such that $\psi(x)=\frac{1}{2} \varphi(h x+x h)$ for all $x \in M$ [6]. In [10] we established the relationship of this Radon Nikodym theorem with the Tomita-Takesaki theory for von Neumann algebras with a separating and cyclic vector. In fact in this paper we showed that from a slight generalization of Sakai's theorem, it follows that the resolvent $(\Delta-\omega)^{-1}$ of the modular operator $\Delta$ associated with a separating and cyclic vector $\xi_{0}$ for $M$, maps the set $M^{\prime} \xi_{0}$ into $M \xi_{0}$ for any $\omega \in C$ with $|\omega|=1$ and $\omega \neq 1$.

Combes has shown [2] that with every faithful normal semi-finite weight $\varphi$ on a von Neumann algebra $M$ is canonically associated a left Hilbert algebra. In this paper we use some of the techniques introduced in $[9,10]$ and the Tomita-Takesaki theory to obtain a generalization of Sakai's Radon Nikodym theorem for weights. If $\psi$ is any weight majorized by $\varphi$ we construct a Radon Nikodym derivative $h \in M$ with $0 \leqq h \leqq 1$. If $\mathscr{L}_{\varphi}$ denotes the subalgebra spanned by the set $\left\{x \in M^{+}, \varphi(x)<\infty\right\}$ we prove that $x h+h x \in \mathscr{M}_{\varphi}$ for any $x$ in a certain $\sigma$-weakly dense *-subalgebra of $M$ and that $\psi(x)=$ $\frac{1}{2} \varphi(h x+x h)$. Moreover we give a counterexample to show that in general we can not expect that $x h+h x \in \mathscr{M}_{\varphi}$ for any $x \in \mathscr{M}_{\varphi}$ so that $\varphi(h x+x h)$ would not even be defined.

If $\psi$ would be invariant with respect to the modular automor- 
phism group associated with $\varphi$ we would obtain that also the Radon Nikodym derivative $h$ would be invariant so that $\varphi(h x)=\varphi(x h)$ for any $x \in \mathscr{H}_{\varphi}$ and our result would follow from the one of Pedersen and Takesaki [5]. However in this case they obtain much stronger results: they show that the equality $\psi(x)=\varphi(h x)$ holds for all $x \in M^{+}$, in some sense, and that a similar result is true even when $\psi$ is not majorized by $\varphi$. So the theorem of Pedersen and Takesaki compares two normal semi-finite weights on the whole of $M^{+}$under the assumption that one is invariant with respect to the modular automorphisms of the other. Our theorem requires no such condition but assumes that $\Psi \leqq \varnothing$ and relates the weights only on a dense subalgebra.

For the theory of von Neumann algebras we refer to $[4,6]$, for the one of left Hilbert algebras to [7, 9], for weights to [1, 8], and for the connection between the last two to $[2,8]$. This work was initiated while I was a guest of Prof. J. Ringrose at the University of Newcastle upon Tyne. It was completed during my stay at the University of Pennsylvania and I would like to express my thanks to Prof. R. V. Kadison for his kind hospitality. I am also indebted to Dr. A. Connes who provided the essential idea (Lemma 2.6) for the counterexample.

2. Some operator equations. Let $\Delta$ be a nonsingular positive self-adjoint operator on a Hilbert space $\mathscr{H}$. In this section we will obtain some operator equations very similar and closely related to those obtained in $[9,10]$. There we expressed $\Delta^{1 / 2}(\Delta-\omega)^{-1}$ for $\omega \in$ $C \backslash[0, \infty)$ as an integral

$$
\Delta^{1 / 2}(\Delta-\omega)^{-1}=\int_{-\infty}^{+\infty} f(t) \Delta^{i t} d t
$$

where $f$ is a nice $L_{1}$-function on $\boldsymbol{R}$, the integral being defined in the strong operator topology. In some sense we also treated the analogue of this relation for the operators $\sigma_{t}: x \in \mathfrak{B}(\mathscr{H}) \rightarrow \Delta^{i t} x \Delta^{-i t}$ instead of $\Delta^{i t}$.

In this paper we are concerned with the operator $(\Delta-1)(\Delta+1)^{-1}$. We also express it in terms of the unitaries $\Delta^{i t}$. The main difference, which turns out to imply the main difficulties in this paper, is that the corresponding function on $\boldsymbol{R}$ is not anymore an $L_{1}$-function. We have to use principle values. We show at the end of this section that in this case the analogue with the $\sigma_{t}$ in general does not define a bounded operator on $\mathfrak{B}(\mathscr{H})$ anymore. And it is precisely this trouble that causes the Radon Nikodym theorem only to hold on a dense subset. 
In this section, a part from $\Delta$, we also fix any element $x_{0} \in \mathfrak{B}(\mathscr{H})$ with $0 \leqq x_{0} \leqq 1$ and we define $h \in \mathfrak{B}(\mathscr{H})$ by

$$
h=\int_{-\infty}^{+\infty} \frac{2}{e^{\pi t}+e^{-\pi t}} \Delta^{i t} x_{0} \Delta^{-i t} d t
$$

in the strong operator topology. It is clear that $h$ is positive. In fact, as

$$
\int_{-\infty}^{+\infty} \frac{2}{e^{\pi t}+e^{-\pi t}} d t=1
$$

we get also $0 \leqq h \leqq 1$. For more details we refer to $[9,10]$. The last equality can e.g. be obtained by the analytic function method of [9.10] or by replacing $\Delta=1$ and $r=1$ in [9, Lemma 4.3]

Notations 2.1. By $\rho$ we denote the real function on $R \backslash\{0\}$ with values

$$
\rho(t)=\frac{2}{e^{\pi t}-e^{-\pi t}} .
$$

The function is odd and has a singularity at $t=0$. For any bounded continuous function $f: R \rightarrow C$ we will write

$$
P \int_{-\infty}^{+\infty} \rho(t) f(t) d t=q
$$

where $q \in \boldsymbol{C}$ when the limit of

$$
\int_{-\infty}^{-\varepsilon} \rho(t) f(t) d t+\int_{\varepsilon}^{\infty} \rho(t) f(t) d t=\int_{\varepsilon}^{\infty} \rho(t)(f(t)-f(-t)) d t
$$

exists when $\varepsilon \rightarrow 0$ and is equal to $q$. In other words $P$ stands for the principle value of the integral. Similar notations will be used for functions $f$ of $\boldsymbol{R}$ to $\mathscr{H}$ or $\mathfrak{B}(\mathscr{H})$ in which case the topology on $\mathscr{H}$ or $\mathfrak{B}(\mathscr{H})$ will be specified.

Lemma 2.2. If $\xi \in \mathscr{D}\left(\Delta^{-1 / 2}\right)$ and $\eta \in \mathscr{D}\left(\Delta^{1 / 2}\right)$ we have

$$
P \int_{-\infty}^{+\infty} \rho(t)\left(\Delta^{i t} x_{0} \Delta^{-i t} \xi, \eta\right) d t=i\left(h \Delta^{-1 / 2} \xi, \Delta^{1 / 2} \eta\right)-i\left(x_{0} \xi, \eta\right) .
$$

Proof. As $\xi \in \mathscr{D}\left(\Delta^{-1 / 2}\right)$ the function $\alpha \in C \rightarrow \Delta^{\alpha} \xi$ is analytic inside and bounded and continuous on the strip $-\frac{1}{2} \leqq \operatorname{Re} \alpha \leqq 0$ [5, Lemma 3.2]. Similarly, as $\eta \in \mathscr{D}\left(\Delta^{1 / 2}\right)$ the function $\alpha \in C \rightarrow \Delta^{\alpha} \eta$ is analytic inside and bounded and continuous on the strip $0 \leqq \operatorname{Re} \alpha \leqq \frac{1}{2}$. The norm topology on $\mathscr{H}$ is considered. A little thought shows that the function 


$$
z \in C \longrightarrow\left(x_{0} \Delta^{-i z} \Delta^{-1 / 2} \xi, \Delta^{-i \bar{z}} \Delta^{1 / 2} \eta\right)
$$

is defined, bounded and continuous on the strip $0 \leqq \operatorname{Im} z \leqq \frac{1}{2}$ and also analytic inside. On the other hand, the function $z \in C \rightarrow 2\left(e^{\pi z}+e^{-\pi z}\right)^{-1}$ is also analytic and continuous on this strip, except at the point $z=i / 2$ where there is a pole with residu $(\pi i)^{-1}$. Moreover this function tends to zero when $z \rightarrow \infty$ on this strip. Therefore by integrating along the boundary we obtain

$$
\begin{aligned}
& \int_{-\infty}^{+\infty} \frac{2}{e^{\pi t}+e^{-\pi t}}\left(x_{0} \Delta^{-i t} \Delta^{-1 / 2} \xi, \Delta^{-i t} \Delta^{1 / 2} \eta\right) d t \\
& \quad=P \int_{-\infty}^{+\infty} \frac{(-2 i)}{e^{\pi t}-e^{-\pi t}}\left(x_{0} \Delta^{-i t} \xi, \Delta^{-i t} \eta\right) d t+\left(x_{0} \xi, \eta\right)
\end{aligned}
$$

and the lemma follows from the definitions of $h$ and of $\rho$.

LEMMA 2.3.

$$
P \int_{-\infty}^{+\infty} \rho(t) \Delta^{i t} d t=i \frac{\Delta-1}{\Delta+1}
$$

in the strong operator topology.

Proof. Take first $\xi \in \mathscr{D}\left(\Delta^{1 / 2}\right)$ and apply the same method as in Lemma 2.2 with the function

$$
z \in C \longrightarrow \frac{2}{e^{\pi z}+e^{-\pi z}} \Delta^{i z} \Delta^{1 / 2} \xi .
$$

We obtain

$$
\int_{-\infty}^{+\infty} \frac{2}{e^{\pi t}+e^{-\pi t}} \Delta^{i t} \Delta^{1 / 2} \xi d t=P \int_{-\infty}^{+\infty} \frac{(-2 i)}{e^{\pi t}-e^{-\pi t}} \Delta^{i t} \xi d t+\xi
$$

in the strong operator topology.

From $[9,10]$ we know that

$$
\frac{2 \Delta^{1 / 2}}{\Delta+1}=\int_{-\infty}^{+\infty} \frac{2}{e^{\pi t}+e^{-\pi t}} \Delta^{i t} d t
$$

in the strong operator topology.

So that

$$
i \frac{2 \Delta}{\Delta+1} \xi=P \int_{-\infty}^{+\infty} \rho(t) \Delta^{i t} \xi d t+i \xi
$$

or

$$
i \frac{\Delta-1}{\Delta+1} \xi=P \int_{-\infty}^{+\infty} \rho(t) \Delta^{i t} \xi d t
$$


Replacing $\Delta$ by $\Delta^{-1}$ we obtain for any $\eta \in \mathscr{D}\left(\Delta^{-1 / 2}\right)$

$$
\begin{aligned}
i \frac{\Delta^{-1}-1}{\Delta^{-1}+1} \eta & =P \int_{-\infty}^{+\infty} \rho(t) \Delta^{-i t} \eta d t \\
& =-P \int_{-\infty}^{+\infty} \rho(t) \Delta^{i t} \eta d t
\end{aligned}
$$

by a change of variable. Again

$$
\frac{\Delta^{-1}-1}{\Delta^{-1}+1}=-\frac{\Delta-1}{\Delta+1}
$$

so that

$$
i \frac{\Delta-1}{\Delta+1} \eta=P \int_{-\infty}^{+\infty} \rho(t) \Delta^{i t} \eta d t
$$

This now holds for any $\eta \in \mathscr{D}\left(\Delta^{1 / 2}\right)$ and $\eta \in \mathscr{D}\left(\Delta^{-1 / 2}\right)$. Therefore by linearity it holds for $\eta \in \mathscr{D}\left(\Delta^{1 / 2}\right)+\mathscr{D}\left(\Delta^{-1 / 2}\right)$.

Finally the formula

$$
1=\frac{1}{1+\Delta^{1 / 2}}+\frac{\Delta^{1 / 2}}{1+\Delta^{1 / 2}}=\frac{1}{1+\Delta^{1 / 2}}+\frac{1}{1+\Delta^{-1 / 2}}
$$

shows that $\mathscr{H}=\mathscr{D}\left(4^{1 / 2}\right)+\mathscr{D}\left(\Delta^{-1 / 2}\right)$ and the proof is complete.

LEMma 2.4. If $\xi \in \mathscr{D}(\log 4)$ then

$$
P \int_{-\infty}^{+\infty} \rho(t) \Delta^{i t} x_{0} \Delta^{-i t} \xi d t
$$

exists. If moreover $\xi \in \mathscr{D}\left(\Delta^{-1 / 2}\right)$ then $h \Delta^{-1 / 2} \xi \in \mathscr{D}\left(\Delta^{1 / 2}\right)$ and

$$
\Delta^{1 / 2} h \Delta^{-1 / 2} \xi-x_{0} \xi=-i P \int_{-\infty}^{+\infty} \rho(t) \Delta^{i t} x_{0} \Delta^{-i t} \xi d t .
$$

Proof. Assume first that $\xi \in \mathscr{D}(\log \Delta)$. Then

$$
\Delta^{i t} x_{0} \Delta^{-i t} \xi=\Delta^{i t} x_{0} \xi+\Delta^{i t} x_{0}\left(\Delta^{-i t}-1\right) \xi \text {. }
$$

From Lemma 2.3 we know that $P \int_{-\infty}^{+\infty} \rho(t) \Delta^{i t} x_{0} \xi d t$ exists. As $\xi \epsilon$ $\mathscr{D}(\log \Delta)$ we have that

$$
\rho(t) \Delta^{i t} x_{0}\left(\Delta^{-i t}-1\right) \xi=\frac{2 t}{e^{\pi t}-e^{-\pi t}} \Delta^{i t} x_{0}\left(\frac{\Delta^{-i t}-1}{t} \xi\right)
$$

is continuous at the point $t=0$ in the norm topology. So it is continuous everywhere and as it tends fast to zero at infinity it is integrable. The combination of the two results yields the first statement of the lemma. To prove the second statement, assume that 
also $\xi \in \mathscr{D}\left(\Delta^{-1 / 2}\right)$ and take any $\eta \in \mathscr{D}\left(\Delta^{1 / 2}\right)$. Then we have

$$
\begin{aligned}
& \left(P \int_{-\infty}^{+\infty} \rho(t) \Delta^{i t} x_{0} \Delta^{-i t} \xi d t, \eta\right) \\
& \quad=P \int_{-\infty}^{+\infty} \rho(t)\left(\Delta^{i t} x_{0} \Delta^{-i t} \xi, \eta\right) d t \\
& \quad=i\left(h \Delta^{-1 / 2} \xi, \Delta^{1 / 2} \eta\right)-i\left(x_{0} \xi, \eta\right)
\end{aligned}
$$

using Lemma 2.2. This shows, as ${\Delta^{1 / 2}}^{\text {is }}$ self-adjoint, that $h \Delta^{-1 / 2} \xi \epsilon$ $\mathscr{D}\left(\Delta^{1 / 2}\right)$ and

$$
\Delta^{1 / 2} h \Delta^{-1 / 2} \xi-x_{0} \xi=-i P \int_{-\infty}^{+\infty} \rho(t) \Delta^{i t} x_{0} \Delta^{-i t} \xi d t .
$$

We will now proceed to show that in general one can not expect Lemma 2.4 to hold for any $\xi \in \mathscr{H}$. This result will provide a counterexample in $\S 4$.

Lemma 2.5. Suppose that for any $\xi \in \mathscr{H}$ there is a vector $y \xi$ such that $y \xi=P \int_{-\infty}^{+\infty} \rho(t) \Delta^{i t} x_{0} \Delta^{-i t} \xi d t$ in the weak topology. Then $y$ is a bounded linear operator on $\mathscr{H}$.

Proof. For any $\xi, \eta \in \mathscr{C}$ we have

$$
\begin{aligned}
(y \xi, \eta) & =P \int_{-\infty}^{+\infty} \rho(t)\left(\Delta^{i t} x_{0} \Delta^{-i t} \xi, \eta\right) d t \\
& =P \int_{-\infty}^{+\infty} \rho(t)\left(\xi, \Delta^{i t} x_{0} \Delta^{-i t} \eta\right) d t \\
& =(\xi, y \eta)
\end{aligned}
$$

where we use the fact that $\rho$ is real and $x_{0}$ self adjoint. Therefore $y$ is symmetric and has a closed extension which is $y$ again because it is everywhere defined. By the closed graph theorem $y$ is continuous.

LEMMA 2.6. There exists a Hilbert space $\mathscr{H}$, a positive nonsingular self-adjoint operator $\Delta$ in $\mathscr{H}$ and a positive operator $x_{0} \epsilon$ $\mathfrak{B}(\mathscr{H})$ such that the map

$$
\xi \in \mathscr{D}(\log \Delta) \longrightarrow P \int_{-\infty}^{+\infty} \rho(t) \Delta^{i t} x_{0} \Delta^{-i t} \xi d t
$$

is not continuous.

Proof. Take $\mathscr{H}=L_{2}(R)$ and let $\Delta$ be defined by

$$
\left(\Delta^{i t} \xi\right)(s)=\xi(s-t) \quad \text { for } \xi \in L_{2}(R) .
$$

Let $x_{0}$ be defined by $\left(x_{0} \xi\right)(s)=g(s) \xi(s)$ where 


$$
g(s)=1 \quad \text { when } s<0
$$

and

$$
g(s)=0 \quad \text { when } s \geqq 0 .
$$

For any $\xi \in L_{2}(R)$ we have

$$
\begin{aligned}
\left(\Delta^{i t} x_{0} \Delta^{-i t} \xi\right)(s) & =\left(x_{0} \Delta^{-i t} \xi\right)(s-t) \\
& =g(s-t)\left(\Delta^{-i t} \xi\right)(s-t) \\
& =g(s-t) \xi(s) .
\end{aligned}
$$

For any $\eta \in L_{2}(R)$ and $\xi \in \mathscr{D}(\log \Delta)$ we have

$$
\begin{aligned}
P \int_{-\infty}^{+\infty} & \rho(t)\left(\Delta^{i t} x_{0} \Delta^{-i t} \xi, \eta\right) d t \\
& =\int_{0}^{\infty} \rho(t)\left(\left(\Delta^{i t} x_{0} \Delta^{-i t}-\Delta^{-i t} x_{0} \Delta^{i t}\right) \xi, \eta\right) d t \\
& =\int_{0}^{\infty} \rho(t)\left(\int_{-\infty}^{+\infty}(g(s-t)-g(s+t)) \xi(s) \overline{\eta(s)} d s\right) d t .
\end{aligned}
$$

It follows from Lemma 2.4 that this integral always exists for any $\eta \in L_{2}(R)$.

If we multiply $\eta$ with a suitable function of modulus one, the integrand would become positive and the integral would still exist. Therefore the integral converges absolutely and we may apply Fubini's theorem. Then

$$
\begin{aligned}
P \int_{-\infty}^{+\infty} & \rho(t)\left(\Delta^{i t} x \Delta^{-i t} \xi, \eta\right) d t \\
& =\int_{-\infty}^{+\infty} \xi(s) \overline{\eta(s)}\left(\int_{0}^{\infty}(g(s-t)-g(s+t)) \rho(t) d t\right) d s \\
& =\int_{-\infty}^{+\infty} \psi(s) \xi(s) \overline{\eta(s)} d s
\end{aligned}
$$

where

$$
\psi(s)=\int_{|s|}^{\infty} \rho(t) d t=\int_{|s|}^{\infty} \frac{2}{e^{\pi t}-e^{-\pi t}} d t .
$$

As $\rho(t)$ behaves like $t^{-1}$ for small $t$, it is not integrable and therefore $\psi$ is not bounded. It is easily seen that in this case the integral cannot be continuous on $\xi \in \mathscr{D}(\log \Delta)$.

Consider the functions

$$
\xi_{n}(t)=\sqrt{n} \exp -(n t)^{2} .
$$

Clearly $\xi_{n}$ is analy tic for $\Delta^{i t}$ and therefore belongs to $\mathscr{D}(\log \Delta)$. Moreover $\left\|\xi_{n}\right\|$ is independent of $n$ while $\int_{-\infty}^{+\infty} \psi(s) \xi_{n}(s) \overline{\eta(s)} d s$ clearly tends 
to $\infty$ because $\psi$ is not bounded at the origin when $\eta$ is e.g. not zero in a neighborhood of $s=0$.

3. The main theorem. Let $\varphi$ be a faithful normal weight on a von Neumann algebra $M$. The set $\mathscr{N}_{\varphi}=\left\{x \in M \mid \varphi\left(x^{*} x\right)<\infty\right\}$ is a $\sigma$-weakly dense left ideal of $M$. The subalgebra $\mathscr{N}_{\varphi}=\mathscr{N}_{\varphi}^{*} \mathscr{N}_{\varphi}$ is spanned by its positive elements $\mathscr{M}_{\varphi}^{+}$and $\mathscr{M}_{\varphi}^{+}=\left\{x \in M^{+} \mid \varphi(x)<\infty\right\}$. Therefore $\varphi$ can be uniquely extended to a linear functional on $\mathscr{M}_{\varphi}$, still denoted by $\varphi$. The set $\mathscr{N}_{\varphi}$ is a pre-Hilbertspace with the scalar product $(x, y)=\varphi\left(y^{*} x\right)$. The completion is denoted by $\mathscr{H}_{\varphi}$. The map $x \in \mathscr{N}_{\varphi} \rightarrow y x \in \mathscr{N}_{\varphi}$ is continuous with respect to the inner product and therefore can be extended to a bounded operator $\pi_{\varphi}(y)$ on $\mathscr{H}_{\varphi}$. It follows that $\pi_{\varphi}$ is a ${ }^{*}$-representation, and from the normality of $\phi$ that $\pi_{\varphi}$ is normal. In fact $\pi_{\varphi}$ is an isomorphism. The subalgebra $\mathscr{N}_{\varphi} \cap \mathscr{N}_{\varphi}^{*}$ turns out to be an achieved left Hilbert algebra with left von Neumann algebra $\pi_{\varphi}(M)$.

For more detailed information and proofs we refer to the works of Combes [1,2] and Takesaki [8]. The results in this section are heavily based on the relationship between weights and left Hilbert algebras as described above.

THEOREM 3.1. Let $\varphi$ be a faithful normal semifinite weight on a von Neumann algebra $M$. There exists a $\sigma$-weakly dense *-subalgebra $M_{0}$ of $M$ such that for any weight $\psi$ on $M$ majorized by $\varphi$ there is an element $h \in M$ with $0 \leqq h \leqq 1$ such that $x h+h x \in \mathscr{M}_{\varphi}$ for any $x \in M_{0}$ and $\psi(x)=\frac{1}{2} \varphi(x h+h x)$.

Proof. Without loss of generality we may assume that $M$ is the left von Neumann algebra $\mathscr{L}(\mathscr{A})$ of an achieved left Hilbert algebra $\mathscr{A}$ and that $\varphi$ is the canonical weight on $\mathscr{L}(\mathscr{A})$. Then for any $\xi$, $\eta \in \mathscr{A}$ we have $\pi\left(\xi^{\sharp} \eta\right) \in \mathscr{A}_{\varphi}$ and

$$
\varphi\left(\pi\left(\xi^{\sharp} \eta\right)\right)=(\eta, \xi) .
$$

Given any other weight $\psi$ such that $\psi(x) \leqq \varphi(x)$ for any $x \in M^{+}$, there exists an element $x_{0}^{\prime} \in M^{\prime}$ such that $0 \leqq x_{0}^{\prime} \leqq 1$ and

$$
\varphi\left(\pi\left(\xi^{\sharp} \xi\right)\right)=\left(x_{0}^{\prime} \xi, \xi\right)
$$

for any $\xi \in \mathscr{A}$. [1].

Define

$$
x_{0}=J x_{0}^{\prime} J
$$

and

$$
h=\int_{-\infty}^{+\infty} \frac{2}{e^{\pi t}+e^{-\pi t}} \Delta^{i t} x_{0} \Delta^{-i t} d t
$$


where $J$ and $\Delta$ are the cannonical involution and the modular operator of $\mathscr{A}$. Then $x_{0}$ and $h$ are in $M^{+}$. For any $\xi \in \mathscr{A} \cap \mathscr{D}(\log \Delta) \subseteq$ $\mathscr{D}\left(\Delta^{1 / 2}\right) \cap \mathscr{D}(\log \Delta)$ we have $J \xi \in \mathscr{D}\left(\Delta^{-1 / 2}\right) \cap \mathscr{D}(\log \Delta)$ as $J \Delta J=\Delta^{-1}$ and $J(\log \Delta) J=-\log \Delta$. Therefore we can apply Lemma 2.4 to obtain that

$$
h \Delta^{-1 / 2} J \xi \in \mathscr{D}\left(\Delta^{1 / 2}\right)
$$

and that

$$
\operatorname{Sh} S \xi=J \Delta^{1 / 2} h \Delta^{-1 / 2} J \xi=J x_{0} J \xi-i P \int_{-\infty}^{+\infty} \rho(t) J \Delta^{i t} x_{0} \Delta^{-i t} J \xi d t .
$$

So

$$
(\operatorname{Sh} S \xi, \xi)=\left(J x_{0} J \xi, \xi\right)-i P \int_{-\infty}^{+\infty} \rho(t)\left(J \Delta^{i t} x_{0} \Delta^{-i t} J \xi, \xi\right) d t .
$$

As $x_{0}=J x_{0}^{\prime} J$ and $x_{0}^{\prime}$ is positive it follows that

$$
\operatorname{Re}(\operatorname{Sh} S \xi, \xi)=\left(J x_{0} J \xi, \xi\right)=\left(x_{0}^{\prime} \xi, \xi\right) .
$$

Next we show that $h S \xi \in \mathscr{A}$. Therefore take $\eta \in \mathscr{A}^{\prime}$ and consider

$$
\pi^{\prime}(\eta) h S \xi=h \pi^{\prime}(\eta) S \xi=h \pi(\xi)^{*} \eta .
$$

Knowing that also $h S \xi \in \mathscr{D}(S)$ we obtain $h S \xi \in \mathscr{A}$ and $\pi(h S \xi)=h \pi(\xi)^{*}$. Then

$$
\begin{aligned}
\left(x_{0}^{\prime} \xi, \xi\right) & =\operatorname{Re}(\operatorname{Sh} S \xi, \xi) \\
& =\operatorname{Re} \varphi\left(\pi(\xi)^{*} \pi(\operatorname{Sh} S \xi)\right) \\
& =\operatorname{Re} \varphi\left(\pi(\xi)^{*} \pi(h S \xi)^{*}\right) \\
& =\operatorname{Re} \varphi\left(\pi(\xi)^{*} \pi(\xi) h\right) .
\end{aligned}
$$

So $\psi\left(\pi\left(\xi^{\sharp} \xi\right)\right)=\frac{1}{2} \varphi\left(\pi\left(\xi^{\sharp} \xi\right) h+h \pi\left(\xi^{\sharp} \xi\right)\right)$.

To complete the proof, observe that for any $\xi \in \mathscr{X}_{0}$, the Tomita algebra associated with $\mathscr{A}$, we have $\xi \in \mathscr{D}(\log \Delta)$. Moreover $\pi\left(\mathscr{A}_{0}\right)$

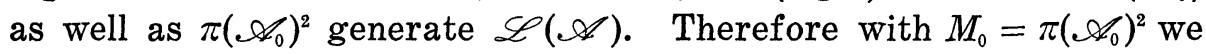
obtain $x h+h x \in \mathscr{N}_{\varphi}$ for $x \in M_{0}$ and $\psi(x)=\frac{1}{2} \varphi(h x+x h)$.

In $\S 4$ we will obtain a counterexample showing that in general one cannot expect that $x h+h x \in \mathscr{K}_{\varphi}$ for any $x \in \mathscr{N}_{\varphi}$, so that certainly $\psi(x)=\frac{1}{2} \varphi(h x+x h)$ will not make sense for some $x \in M^{+}$.

Under some conditions we can show that $h$ is unique. Suppose e.g. that $h_{1} \in M$ with $h_{1}=h_{1}^{*}$ and satisfies the properties $h_{1} \mathscr{X _ { 0 }} \cong \mathscr{A}$ and $\psi(x)=\frac{1}{2} \varphi\left(h_{1} x+x h_{1}\right)$ for all $x \in \pi\left(\mathscr{A}_{0}\right)^{2}$. Then we can show that $h_{1}=h$. Indeed for any $\xi \in \mathscr{A}_{0}$ we have

$$
\begin{aligned}
\psi\left(\pi\left(\xi^{\sharp} \xi\right)\right) & =\frac{1}{2} \varphi\left(\pi\left(\xi^{\sharp} \xi\right) h_{1}+h_{1} \pi\left(\xi^{\sharp} \xi\right)\right) \\
& =\operatorname{Re}\left(\operatorname{Sh}_{1} S \xi, \xi\right) \\
& =\operatorname{Re}(\operatorname{Sh} S \xi, \xi) .
\end{aligned}
$$


When we put $a=J\left(h-h_{1}\right) J$ then

$$
\left(a \Delta^{1 / 2} \xi, \Delta^{-1 / 2} \xi\right)=-\left(a \Delta^{-1 / 2} \xi, \Delta^{1 / 2} \xi\right)
$$

and by linearity

$$
\left(a \Delta^{1 / 2} \eta, \Delta^{-1 / 2} \xi\right)=-\left(a \Delta^{-1 / 2} \eta, \Delta^{1 / 2} \xi\right)
$$

for any $\xi, \eta \in \mathscr{A}$. Clearly $\Delta^{-1 / 2} \mathscr{A}_{0}=\mathscr{A}_{0}$ and therefore $\left(\Delta^{1 / 2}+\Delta^{-1 / 2}\right) \mathscr{A}_{0}=$ $(1+\Delta) \mathscr{X}_{0}$ is dense [7]. As in [9, Lemma 3.3] it follows that the above relation then holds for any $\xi, \eta \in \mathscr{D}\left(\Delta^{1 / 2}\right) \cap \mathscr{D}\left(\Delta^{-1 / 2}\right)$ and in a similar way that $a=0$.

In the case where $\psi$ is a normal positive linear functional one can obtain a better result, and it is not necessary to use the results of $\S 2$.

THEOREM 3.2. Let $\phi$ be a faithful normal semi-finite weight on a von Neumann algebra $M$. For every normal positive linear functional $f$ on $M$ majorized by $\varphi$ there is a unique element $h \in \mathscr{M}_{\varphi}$ with $0 \leqq h \leqq 1$ such that

$$
f(x)=\frac{1}{2} \varphi(h x+x h) \quad \text { for all } x \in \mathscr{N}_{\varphi} \cap \mathscr{N}_{\varphi}^{*} .
$$

Proof. Again we may assume that $M$ is the left von Neumann algebra of an achieved left Hilbert algebra $\mathscr{A}$, and that $\varphi$ is the canonical weight on $M$. As $f$ is now continuous there is a vector $\alpha \in \mathscr{H}$ and an operator $x_{0}^{\prime} \in M^{\prime}$ with $0 \leqq x_{0}^{\prime} \leqq 1$ such that $f(x)=$ $(x \alpha, \alpha)$ for all $x \in M$ and $x_{0}^{\prime 1 / 2} \eta=\pi(\eta) \alpha$ for all $\eta \in \mathscr{A}$. [1, 2]. So $\alpha \in \mathscr{A}^{\prime}$ and $\pi^{\prime}(\alpha)=x_{0}^{\prime 1 / 2}$. Then $\beta=x_{0}^{\prime 1 / 2} \alpha \in \mathscr{A}^{\prime}$ and $F \beta=\beta$ and $\pi^{\prime}(\beta)=x_{0}^{\prime}$.

Put $\xi_{0}=2 /(\Delta+1) \beta$, then $\xi_{0} \in \mathscr{A}$. [7,9]. From the fact that $F \beta=\beta$ and $J \Delta J=\Delta^{-1}$ it follows that $S \xi_{0}=\xi_{0}$.

For any $\xi \in \mathscr{A}$ we have

$$
\begin{aligned}
f(\pi(\xi)) & =(\pi(\xi) \alpha, \alpha) \\
& =\left(x_{0}^{1 / 2} \xi, \alpha\right) \\
& =\left(\xi, x_{0}^{1 / 2} \alpha\right) \\
& =(\xi, \beta) \\
& =\frac{1}{2}\left(\xi, \Delta \xi_{0}\right)+\frac{1}{2}\left(\xi, \xi_{0}\right) \\
& =\frac{1}{2}\left(\xi, F S \xi_{0}\right)+\frac{1}{2}\left(\xi, \xi_{0}\right) \\
& =\frac{1}{2}(\xi, S \xi)+\frac{1}{2}\left(\xi, \xi_{0}\right) \\
& =\frac{1}{2} \varphi\left(\pi(\xi) \pi\left(\xi_{0}\right)+\pi\left(\xi_{0}\right) \pi(\xi)\right) .
\end{aligned}
$$

As $\pi(\mathscr{A})=\mathscr{N}_{\varphi} \cap \mathscr{N}_{\varphi}^{*}$ we get for any $x \in \mathscr{N}_{\varphi} \cap \mathscr{N}_{\varphi}^{*}$ that $f(x)=$ $\frac{1}{2} \varphi(x h+h x)$ where $h=\pi\left(\xi_{0}\right)$.

To continue the proof of our theorem we show that $h \in \mathscr{L}_{\varphi}$ and 
that $0 \leqq h \leqq 1$.

As $h=\pi\left(\xi_{0}\right)$ and $\xi_{0} \in \mathscr{A}$ with $S \xi_{0}=\xi_{0}$ it is clear that $h \in \mathscr{N}_{\varphi} \cap \mathscr{N}_{\varphi}^{*}$ and $h=h^{*}$.

Now

$$
\begin{aligned}
\xi_{0} & =J \Delta^{1 / 2} \frac{2}{\Delta+1} \beta \\
& =\int_{-\infty}^{+\infty} \frac{2}{e^{\pi t}+e^{-\pi t}} \Delta^{i t} J \beta d t \quad[9,10] .
\end{aligned}
$$

It follows that

$$
\begin{aligned}
h=\pi\left(\xi_{0}\right) & =\int_{-\infty}^{+\infty} \frac{2}{e^{\pi t}+e^{-\pi t}} \pi\left(\Delta^{i t} J \beta\right) d t \\
& =\int_{-\infty}^{+\infty} \frac{2}{e^{\pi t}+e^{-\pi t}} \Delta^{i t} J \pi^{\prime}(\beta) J \Delta^{-i t} d t \\
& =\int_{-\infty}^{+\infty} \frac{2}{e^{\pi t}+e^{-\pi t}} \Delta^{i t} J x_{0}^{\prime} J \Delta^{-i t} d t
\end{aligned}
$$

As $0 \leqq x_{0}^{\prime} \leqq 1$ it again follows that $0 \leqq h \leqq 1$. Moreover

$$
\varphi(h)=\varphi\left(J x_{0}^{\prime} J\right)=(J \alpha, J \alpha)<\infty
$$

by the invariance and the normality of $\varphi$. Therefore $h \in \mathscr{H}_{\varphi}$.

To finish the proof we must show that $h$ is unique. So suppose $h_{1} \in \mathscr{M}_{\varphi}, h_{1}=h_{1}^{*}$ and

$$
f(x)=\frac{1}{2} \varphi\left(h_{1} x+x h_{1}\right) \quad \text { for any } x \in \mathscr{N}_{\varphi} \cap \mathscr{N}_{\varphi}^{*} .
$$

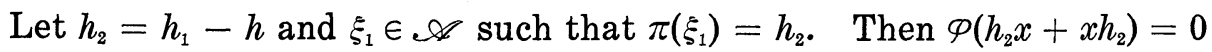
for any $x \in \mathscr{N}_{\varphi} \cap \mathscr{N}_{\varphi}^{*}$ and therefore $\left(\xi, \xi_{1}\right)+\left(\xi_{1}, S \xi\right)=0$ for any $\xi \in \mathscr{A}$. As $\xi_{1}=S \xi_{1}$ this would imply $-\left(\xi, \xi_{1}\right)=\left(S \xi_{1}, S \xi\right)=0$ for all $\xi \in \mathscr{A}$ and $S \xi_{1} \in \mathscr{D}(F)$ with $F S \xi_{1}=-\xi_{1}$. This would imply $\xi_{1}=0$ as $\Delta=F S$ is positive.

In the next section we will give an example to show that also in this case one can not hope that in general $f(x)=\frac{1}{2} \varphi(h x+x h)$ for all $x \in M$.

4. Two counterexamples. In this section we will obtain counterexamples to show that the Theorem 3.1 and 3.2 are the best possible in some sense. In connection with Theorem 3.1 we will construct two faithful normal semifinite weights $\psi$ and $\varphi$ on a von Neumann algebra $M$ with $\psi \leqq \varphi$ and an element $x \in \mathscr{M}_{\varphi}$ such that $h x+x h \notin \mathscr{L}_{\varphi}$ where $h$ is the Radon Nikodym derivative. This will show that in general one can not expect Theorem 3.1 to hold for every $x \in \mathscr{L}_{\varphi}$. In the case of Theorem 3.2 we will show that there is a faithful 
normal semi-finite weight $\varphi$ on a von Neumann algebra $M$ and an $x \in M$ such that $x h+h x \notin \mathscr{L}_{\varphi}$ for any $h \in \mathscr{L}_{\varphi}$. Therefore Theorem 3.2 will not hold for all $x \in M$ in general.

Let $\mathscr{K}$ be a Hilbert space, and $k$ a positive nonsingular self adjoint operator in $\mathscr{K}$. Let $x_{0}$ be any positive operator in $\mathscr{E}(\mathscr{K})$. As before define

$$
h=\int_{-\infty}^{+\infty} \frac{2}{e^{\pi t}+e^{-\pi t}} k^{i t} x_{0} k^{-i t} d t .
$$

The following lemma is closely related to Lemma 2.5.

LEMMA 4.1. With the above notations, suppose that for any $\xi \epsilon$ $\mathscr{D}\left(k^{-1 / 2}\right)$ we have $h k^{-1 / 2} \xi \in \mathscr{D}\left(k^{1 / 2}\right)$ and for any $\eta \in \mathscr{D}\left(k^{1 / 2}\right)$ we have $h k^{1 / 2} \eta \in \mathscr{D}\left(k^{-1 / 2}\right)$ then $\xi \in \mathscr{D}(\log k) \rightarrow P \int_{-\infty}^{+\infty} \rho(t) k^{i t} x_{0} k^{-i t} \xi d t$ is continuous.

Proof. Define two operators $a_{1}$ and $a_{2}$ by

$$
\begin{array}{ll}
a_{1} \xi=k^{1 / 2} h k^{-1 / 2} \xi-x_{0} \xi & \text { for } \xi \in \mathscr{D}\left(k^{-1 / 2}\right) \\
a_{2} \xi=-k^{-1 / 2} h k^{1 / 2} \xi+x_{0} \xi & \text { for } \xi \in \mathscr{D}\left(k^{1 / 2}\right) .
\end{array}
$$

If $\xi \in \mathscr{D}\left(k^{-1 / 2}\right) \cap \mathscr{D}\left(k^{1 / 2}\right)$ then $\xi \in \mathscr{D}(\log k)$ and

$$
a_{1} \xi=-i P \int_{-\infty}^{+\infty} \rho(t) k^{i t} x_{0} k^{-i t} \xi d t
$$

by Lemma 2.4 .

Similarly, by replacing $k$ by $k^{-1}$ we obtain

$$
-a_{2} \xi=-i P \int_{-\infty}^{+\infty} \rho(t) k^{-i t} x_{0} k^{i t} \xi d t,
$$

(Remark that $h$ remains unchanged.) And by a change of variable

$$
a_{1} \xi=a_{2} \xi \text {. }
$$

As $\mathscr{K}=\mathscr{D}\left(k^{1 / 2}\right)+\mathscr{D}\left(k^{-1 / 2}\right)$ and $a_{1} \xi=a_{2} \xi$ for $\xi \in \mathscr{D}\left(k^{1 / 2}\right) \cap \mathscr{D}\left(k^{-1 / 2}\right)$ we can define an operator $a$ on $\mathscr{K}$ by $a\left(\xi_{1}+\xi_{2}\right)=a_{1} \xi_{1}+a_{2} \xi_{2}$ where $\xi_{1} \in \mathscr{D}\left(k^{-1 / 2}\right)$ and $\xi_{2} \in \mathscr{D}\left(k^{1 / 2}\right)$.

We will now prove that $a$ is continuous by showing that the restriction of $-a$ to $\mathscr{D}\left(k^{1 / 2}\right) \cap \mathscr{D}\left(k^{-1 / 2}\right)$ is contained in the adjoint of $a$ so that $a$ is closed and everywhere defined.

So take

$$
\eta \in \mathscr{D}\left(k^{1 / 2}\right) \cap \mathscr{D}\left(k^{-1 / 2}\right), \quad \xi \in \mathscr{D}\left(k^{-1 / 2}\right)
$$

then 


$$
\begin{aligned}
(-a \eta, \xi) & =\left(-a_{2} \eta, \xi\right) \\
& =\left(k^{-1 / 2} h k^{1 / 2} \eta, \xi\right)-\left(x_{0} \eta, \xi\right) \\
& =\left(h k^{1 / 2} \eta, k^{-1 / 2} \xi\right)-\left(x_{0} \eta, \xi\right) \\
& =\left(k^{1 / 2} \eta, h k^{-1 / 2} \xi\right)-\left(x_{0} \eta, \xi\right) \\
& =\left(\eta, k^{1 / 2} h k^{-1 / 2} \xi\right)-\left(\eta, x_{0} \xi\right) \\
& =\left(\eta, a_{1} \xi\right)=(\eta, a \xi) .
\end{aligned}
$$

Similarly $(-a \eta, \xi)=(\eta, a \xi)$ for $\xi \in \mathscr{D}\left(k^{1 / 2}\right)$ and therefore for all $\xi \in \mathscr{K}$. Finally take $\xi \in \mathscr{D}\left(k^{-1 / 2}\right) \cap \mathscr{D}(\log k)$, then

$$
a \xi=a_{1} \xi=-i P \int_{-\infty}^{+\infty} \rho(t) k^{i t} x_{0} k^{-i t} \xi d t
$$

and similarly for $\xi \in \mathscr{D}\left(k^{1 / 2}\right) \cap \mathscr{D}(\log k)$.

As again

$$
\mathscr{D}(\log k)=\mathscr{D}(\log k) \cap \mathscr{D}\left(k^{-1 / 2}\right)+\mathscr{D}(\log k) \cap \mathscr{D}\left(k^{1 / 2}\right) .
$$

The equality holds for any $\xi \in \mathscr{D}(\log k)$. Therefore the lemma follows from the continuity of $a$.

If we combine the previous result with Lemma 2.6 we get, replacing $k$ by $k^{-1}$ if necessary:

LEMMA 4.2. There exists a Hilbert space $\mathscr{K}$, a positive nonsingular self adjoint operator $k$ in $\mathscr{K}$, an element $x_{0} \in \mathfrak{B}(\mathscr{K})$ with $0 \leqq x_{0} \leqq 1$ and a vector $\xi_{0} \in \mathscr{D}\left(k^{-1 / 2}\right)$ such that $h k^{-1 / 2} \xi_{0} \notin \mathscr{D}\left(\mathscr{K}^{1 / 2}\right)$ where

$$
h=\int_{-\infty}^{+\infty} \frac{2}{e^{\pi t}+e^{-\pi t}} k^{i t} x_{0} k^{-i t} d t .
$$

In what follows $\mathscr{K}, k, x_{0}$ and $\xi_{0}$ will be objects satisfying the conditions of this lemma.

Let $M=\mathfrak{B}(\mathscr{K})$ and let $\varphi$ be the faithful normal weight $\operatorname{Tr}(k \cdot)$ as defined in [5]. We will consider the rank one operators in $M$ and we will use the tensor product notation. So for any $\xi, \eta \in \mathscr{K}$ the operator $\xi \otimes \eta$ is defined by

$$
(\xi \otimes \eta) \eta_{1}=\left(\eta_{1}, \eta\right) \xi \quad \text { for } \eta_{1} \in \mathscr{K} .
$$

Lemma 4.3. Let $\xi, \eta \in \mathscr{K}$. Then $\xi \otimes \eta \in \mathscr{M}_{\varphi}$ iff $\xi, \eta \in \mathscr{D}\left(k^{1 / 2}\right)$.

Proof. Take first $\xi_{1} \in \mathscr{K}$ and $\eta \in D\left(k^{1 / 2}\right)$ and put $x=\xi_{1} \otimes \eta$. Then $x^{*}=\eta \otimes \xi_{1}$ and $x^{*} x=\left(\xi_{1}, \xi_{1}\right) \eta \otimes \eta$. As in [5] let $k_{\varepsilon}=k(1+\varepsilon k)^{-1}$. Then $k_{\varepsilon} x^{*} x=\left(\xi_{1}, \xi_{1}\right) k_{\varepsilon} \eta \otimes \eta$ and $\operatorname{Tr} k_{\varepsilon}\left(x^{*} x\right)=\left(\xi_{1}, \xi_{1}\right)\left(k_{\varepsilon} \eta, \eta\right)$. By definition 


$$
\begin{aligned}
\varphi\left(x^{*} x\right) & =\sup _{\varepsilon>0} \operatorname{Tr}\left(k_{\varepsilon} x^{*} x\right) \\
& =\left(\xi_{1}, \xi_{1}\right) \sup _{\varepsilon>0}\left(k_{\varepsilon} \eta, \eta\right) \\
& =\left(\xi_{1}, \xi_{1}\right) \sup _{\varepsilon>0}\left((1+\varepsilon k)^{-1} k^{1 / 2} \eta, k^{1 / 2} \eta\right) .
\end{aligned}
$$

So $\varphi\left(x^{*} x\right)=\left(\xi_{1}, \xi_{1}\right)\left(k^{1 / 2} \eta, k^{1 / 2} \eta\right)$ and $x \in \mathscr{N}_{\varphi}$.

So if $\xi, \eta \in \mathscr{D}\left(k^{1 / 2}\right)$ then

$$
\xi \otimes \eta=\left(\xi_{1} \otimes \xi\right)^{*}\left(\xi_{1} \otimes \eta\right) \quad \text { when }\left\|\xi_{1}\right\|=1
$$

so that $\xi \otimes \eta \in \mathscr{N}_{\varphi}^{*} \mathscr{N}_{\varphi}=\mathscr{M}_{\varphi}$.

Conversely suppose $\xi \otimes \eta \in \mathscr{K}_{\varphi} . \quad$ Then $\xi \otimes \eta \in \mathscr{N}_{\varphi}$ and the previous computation shows that

$$
\sup _{\varepsilon>0}\left(k_{\varepsilon} \eta, \eta\right)<\infty
$$

This clearly implies that $\eta \in \mathscr{D}\left(k^{1 / 2}\right)$. On the other hand $\mathscr{L}_{\varphi} \subseteq \mathscr{N}_{\varphi}^{*}$ so that $\eta \otimes \xi \in \mathscr{N}_{\varphi}$ and $\xi \in \mathscr{D}\left(k^{1 / 2}\right)$.

THEOREM 4.4. There exist a von Neumann algebra $M$, two faithful normal semi-finite weights $\psi$ and $\varphi$ on $M$ with $\psi \leqq \varphi$, and an element $x \in \mathscr{K}_{\varphi}$ such that $h x+x h \notin \mathscr{L}_{\varphi}$ where $h$ is the Radon Nikodym derivative obtained in Theorem 3.1.

Proof. Let $M$ and $\varphi$ be as before. Consider elements $x_{0} \in \mathfrak{B}(\mathscr{K})$ and $\xi_{0} \in \mathscr{K}$ as in Lemma 4.2 .

Replacing $x_{0}$ by $\frac{1}{2}+\frac{1}{2} x_{0}$ we may assume that $\frac{1}{2} \leqq x_{0} \leqq 1$. Indeed this would induce the replacement of $h$ by $\frac{1}{2}+\frac{1}{2} h$ and $\left(\frac{1}{2}+\frac{1}{2} h\right) k^{-1 / 2} \xi_{0}=$ $\frac{1}{2} k^{-1 / 2} \xi_{0}+\frac{1}{2} h k^{-1 / 2} \xi_{0} \notin \mathscr{D}\left(k^{1 / 2}\right)$ as $h k^{-1 / 2} \xi_{0} \notin \mathscr{D}\left(k^{1 / 2}\right)$. This little modification will make sure that our weight $\psi$ will be normal and faithful.

Let $\xi_{1}=k^{-1 / 2} \xi_{0}$, then $\xi_{1} \in \mathscr{D}\left(k^{1 / 2}\right)$ but $h \xi_{1} \notin \mathscr{D}\left(k^{1 / 2}\right)$. Take also $\eta_{0} \in$ $\mathscr{D}\left(k^{-1 / 2}\right) \cap \mathscr{D}(\log k)$, and put $\eta_{1}=k^{-1 / 2} \eta_{0}$. Then $\eta_{1} \in \mathscr{D}\left(k^{1 / 2}\right)$ and $h \eta_{1} \in$ $\mathscr{D}\left(k^{1 / 2}\right)$.

If $x=\eta_{1} \otimes \xi_{1}$ then clearly by Lemma 4.3 we have $x \in \mathscr{M}_{\varphi}, h x=$ $h \eta_{1} \otimes \xi_{1} \in \mathscr{M}_{\varphi}$ while $x h=\eta_{1} \otimes h \xi_{1} \notin \mathscr{M}_{\varphi}$ and therefore $h x+x h \notin \mathscr{M}_{\varphi}$. Therefore to prove the theorem it will be sufficient to find a faithful normal semi-finite weight $\psi$ on $M$ such that $\psi \leqq \varphi$ and $h$ is the Radon Nikodym derivative.

For this purpose we assume again that $M$ is identified with the left von Neumann algebra $\mathscr{L}(\mathscr{A})$ of an achieved left Hilbert algebra $\mathscr{A}$ and that $\phi$ is the canonical weight on $\mathscr{L}(\mathscr{A})$. If $J$ is the canonical involution then $\psi$ defined on $M^{+}$by $\psi(x)=\left(J x_{0} J \xi, \xi\right)$ when $x=\pi\left(\xi^{\sharp} \xi\right)$ with $\xi \in \mathscr{A}$ and $\psi(x)=\infty$ otherwise, defines a weight on $M$. The proof is essentially the same as for the canonical weight [2]. 
It is clear that $\frac{1}{2} \varphi \leqq \psi \leqq \varphi$ as $\frac{1}{2} \leqq x_{0} \leqq 1$ and that $h$ is the Radon Nikodym derivative since the modular automorphisms are given by $\sigma_{t}(x)=k^{i t} x k^{-i t}$ [5, Theorem 4.6]. It is also clear that $\Psi$ is faithful and semi-finite. Therefore it remains to show that $\Psi$ is normal.

Suppose we can show that $x_{0}$ is the upper bound of an increasing net of positive elements $\left\{x_{\alpha}\right\}$ in $\mathscr{L}_{\varphi}^{+}$. For any $\alpha$ we have $x_{\alpha}^{1 / 2} \in \mathscr{N}_{\varphi} \cap$ $\mathscr{N}_{\varphi}^{*}=\pi(\mathscr{A})$ so there exists a vector $\xi_{\alpha} \in \mathscr{A}$ such that $x_{\alpha}^{1 / 2}=\pi\left(\xi_{\alpha}\right)$.

Then

$$
\begin{aligned}
\Psi\left(\pi\left(\xi^{\sharp} \xi\right)\right) & =\left(J x_{0} J \xi, \xi\right) \\
& =\sup _{\alpha}\left(J x_{\alpha} J \xi, \xi\right) \\
& =\sup _{\alpha}\left(x_{\alpha}^{1 / 2} J \xi, x_{\alpha}^{1 / 2} J \xi\right) \\
& =\sup _{\alpha}\left(\pi^{\prime}(J \xi) \xi_{\alpha}, \pi^{\prime}(J \xi) \xi_{\alpha}\right) \\
& =\sup _{\alpha}\left(\pi\left(\xi^{\sharp} \xi\right) J \xi_{\alpha}, J \xi_{\alpha}\right) .
\end{aligned}
$$

It follows that $\psi$ is the upper envelope on $\mathscr{M}_{\varphi}^{+}$of normal positive linear functionals majorized by $\psi$ on $\mathscr{M}_{\varphi}^{+}$. On the other hand $\phi$ is normal and so is the upper envelope of normal positive linear functionals on $M^{+}$. As $\frac{1}{2} \varphi \leqq \psi$ the supremium of the values of the normal positive linear functionals majorized by $\Psi$ in $M^{+} \mid \mathscr{A}_{\varphi}^{+}$is $\infty$. Therefore $\psi$ is the upper envelope on all of $M^{+}$of normal positive linear functionals, so that $\psi$ is normal.

It remains to show that $x_{0}$ is the upper bound of an increasing net of positive elements $\left\{x_{\alpha}\right\}$ in $\mathscr{H}_{\varphi}^{+}$.

Consider the left ideal $\mathscr{N}_{0}$ of $M$ generated by the rank one operators $\xi_{1} \otimes x_{0}^{-1 / 2} \xi_{2}$ where $\xi_{2} \in \mathscr{D}\left(k^{1 / 2}\right)$ and $\xi_{1} \in \mathscr{K}$. Those operators are clearly norm dense in the finite rank operators and hence $\mathscr{N}_{0}$ is $\sigma$-weakly dense. By [3, Lemma 2.3] there is an increasing net $x_{\alpha}$ in $\left(\mathscr{N}_{0}^{*} \mathscr{N}_{0}\right)^{+}$with supremum 1.

Then $x_{0}=\sup _{\alpha} x_{0}^{1 / 2} \times_{\alpha} x_{0}^{1 / 2}$ and as $x_{0}^{1 / 2} \mathscr{N}_{0}^{*} \leqq \mathscr{N}_{\varphi}^{*}$ we have $x_{0} \frac{1}{2} \times_{\alpha} x_{0}^{1 / 2} \epsilon$ $\mathscr{N}_{\varphi}^{*} \mathscr{N}_{\varphi}=\mathscr{L}_{\varphi}$.

Finally we turn our attention to Theorem 3.2.

THEOREM 4.5. There exists a von Neumann algebra $M$, and a faithful normal semifinite weight $\phi$ on $M$ and an element $x \in M$ such that $x h+h x \notin \mathscr{M}_{\varphi}$ for any $h \in \mathscr{M}_{\varphi}$. So in general Theorem 3.2 will not hold for all $x \in M$.

Proof. Let $M$ and $\varphi$ be as in Theorem 4.4. Take $\xi \in \mathscr{D}\left(k^{1 / 2}\right)$ and $\eta \notin \mathscr{D}\left(k^{1 / 2}\right)$ and put $x=\xi \otimes \eta$. Then $x \in \mathscr{N}_{\varphi}^{*}$ so that $x h \in \mathscr{M}_{\varphi}$ because of Lemma 4.3. Therefore $h x+x h \notin \mathscr{M}_{\varphi}$. 


\section{REFERENCES}

1. F. Combes, Poids sur une C*-algèbre, J. Math. Pures et Appl., 47 (1968), 57-100.

2. —_, Poids associé à une algèbre hilbertienne à gauche, Compositio Math., 23 (1971), 49-77.

3. - Poids et Espérance conditionelles dans les algèbres de von Neumann, Bull. Soc. Math. France, 99 (1971), 73-112.

4. J. Dixmier, Les algêbres d'operateurs dans l'espace hilbertien, Gauthiers Villars, Paris, 1969.

5. G. K. Pedersen and M. Takesaki, The Radon Nikodym theorem for von Neumann algebras, Acta Mathematica, 130 (1973), 53-87.

6. S. Sakai, $C^{*}$-algebras and $W^{*}$-algebras, Ergebnisse der Mathematik und ihrer Grenzgebiete, Band 60., Springer Verlag, Berlin Heidelberg New York, 1971.

7. M. Takesaki, Tomita's theory of modular Hilbert algebras and its applications, Springer lecture notes in mathematics No. 128. Springer Verlag, Berlin Heidelberg New York 1970.

8. - U.C.L.A. Lecture notes on operator algebras.

9. A. Van Daele, $A$ new approach to the Tomita-Takesaki theory of generalized Hilbert Algebras, J. Funct. Analysis, 15 (1974), 378-393.

10. - The Tomita-Takesaki theory for von Neumann algebras with a separating and cyclic vector, To appear in the proceedings of the Varenna conference on mathematical physics, 1973.

Received May 13, 1974. This work was partly supported by NSF grant GP-28976.

ON LEAVE FROM the UNIVERSity OF LeUVEN, BeLgiUm

Permanent address: Department of Mathematics, University of Leuven, Celestijnenlaan $200 \mathrm{~B}, \mathrm{~B}-3030$ Heverlee, Belgium. 


\section{PACIFIC JOURNAL OF MATHEMATICS}

\section{EDITORS}

RICHARD ARENS (Managing Editor)

University of California

Los Angeles, California 90024

\author{
R. A. Beaumont \\ University of Washington \\ Seattle, Washington 98105
}

\section{J. DugundjI}

Department of Mathematics University of Southern California Los Angeles, California 90007

D. Gilbarg and J. Milgram

Stanford University

Stanford, California 94305

\section{ASSOCIATE EDITORS}
E. F. BECKENBACH
B. H. NeumanN
F. WOLF
K. YoshidA

\section{SUPPORTING INSTITUTIONS}

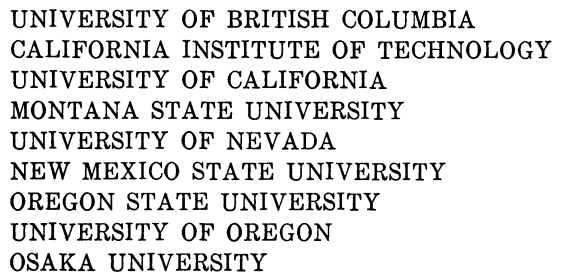

UNIVERSITY OF BRITISH COLUMBIA CALIFORNIA INSTITUTE OF TECHNOLOGY UNIVERSITY OF CALIFORNIA MONTANA STATE UNIVERSITY UNIVERSITY OF NEVADA NEW MEXICO STATE UNIVERSITY OSAKA UNIVERSITY OREGON STATE UNIVERSITY UNIVERSITY OF OREGON

\author{
UNIVERSITY OF SOUTHERN CALIFORNIA \\ STANFORD UNIVERSITY \\ UNIVERSITY OF TOKYO \\ UNIVERSITY OF UTAH \\ WASHINGTON STATE UNIVERSITY \\ UNIVERSITY OF WASHINGTON \\ AMERICAN MATHEMATICAL SOCIETY
}

The Supporting Institutions listed above contribute to the cost of publication of this Journal, but they are not owners or publishers and have no responsibility for its content or policies.

Mathematical papers intended for publication in the Pacific Journal of Mathematics should be in typed form or offset-reproduced, (not dittoed), double spaced with large margins. Please do not use built up fractions in the text of your manuscript. You may however, use them in the displayed equations. Underline Greek letters in red, German in green, and script in blue. The first paragraph or two must be capable of being used separately as a synopsis of the entire paper. Items of the bibliography should not be cited there unless absolutely necessary, in which case they must be identified by author and Journal, rather than by item number. Manuscripts, in triplicate, may be sent to any one of the editors. Please classify according to the scheme of Math. Reviews, Index to Vol. 39. All other communications should be addressed to the managing editor, or Elaine Barth, University of California, Los Angeles, California, 90024.

The Pacific Journal of Mathematics expects the author's institution to pay page charges, and reserves the right to delay publication for nonpayment of charges in case of financial emergency.

100 reprints are provided free for each article, only if page charges have been substantially paid. Additional copies may be obtained at cost in multiples of 50 .

The Pacific Journal of Mathematics is issued monthly as of January 1966. Regular subscription rate: $\$ 72.00$ a year $(6$ Vols., 12 issues). Special rate: $\$ 36.00$ a year to individual members of supporting institutions.

Subscriptions, orders for back numbers, and changes of address should be sent to Pacific Journal of Mathematics, 103 Highland Boulevard, Berkeley, California, 94708.

PUBLISHED BY PACIFIC JOURNAL OF MATHEMATICS, A NON-PROFIT CORPORATION

Printed at Kokusai Bunken Insatsusha (International Academic Printing Co., Ltd.), 8-8, 3-chome, Takadanobaba, Shinjuku-ku, Tokyo 160, Japan. 


\section{Pacific Journal of Mathematics}

\section{Vol. 61, No. 2 December, 1975}

Graham Donald Allen, Francis Joseph Narcowich and James Patrick Williams, An operator version of a theorem of Kolmogorov .......................

Joel Hilary Anderson and Ciprian Foias, Properties which normal operators share with normal derivations and related operators . . . . . . . . . . . . . . . . . . . . .

Constantin Gelu Apostol and Norberto Salinas, Nilpotent approximations and

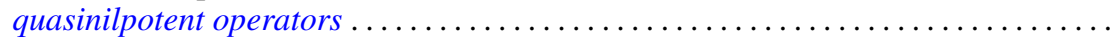

James M. Briggs, Jr., Finitely generated ideals in regular $F$-algebras . . . . . . . . . . .

Frank Benjamin Cannonito and Ronald Wallace Gatterdam, The word problem and power problem in 1-relator groups are primitive recursive ..................

Clifton Earle Corzatt, Permutation polynomials over the rational numbers ...........

L. S. Dube, An inversion of the $S_{2}$ transform for generalized functions . . . . . . . . . . William Richard Emerson, Averaging strongly subadditive set functions in unimodular

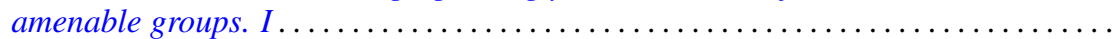

Barry J. Gardner, Semi-simple radical classes of algebras and attainability of

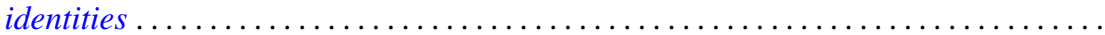

Irving Leonard Glicksberg, Removable discontinuities of A-holomorphic functions ....

Fred Halpern, Transfer theorems for topological structures . . . . . . . . . . . . . . .

H. B. Hamilton, T. E. Nordahl and Takayuki Tamura, Commutative cancellative

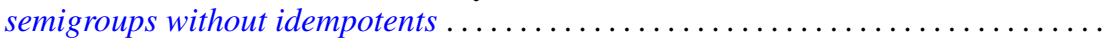

Melvin Hochster, An obstruction to lifting cyclic modules .....................

Alistair H. Lachlan, Theories with a finite number of models in an uncountable power

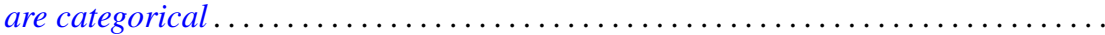

Kjeld Laursen, Continuity of linear maps from $C^{*}$-algebras . . . . . . . . . . . . .

Tsai Sheng Liu, Oscillation of even order differential equations with deviating arguments ....

Jorge Martinez, Doubling chains, singular elements and hyper- $Z$

Mehdi Radjabalipour and Heydar Radjavi, On the geometry of num Thomas I. Seidman, The solution of singular equations, I. Linear equations in Hilbert

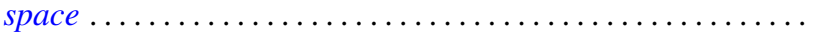

R. James Tomkins, Properties of martingale-like sequences ......

Alfons Van Daele, A Radon Nikodým theorem for weights on von Neumann

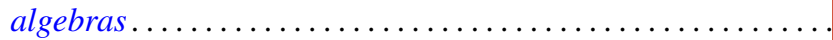

Kenneth S. Williams, On Euler's criterion for quintic nonresidues

Scott Andrew Wolpert, Noncompleteness of the Weil-Petersson metric for Teichmüller

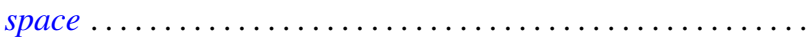

Volker Wrobel, Some generalizations of Schauder's theorem in locally convex

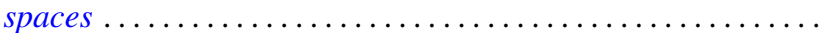

Kelly Denis McKennon, Corrections to: "Multipliers of type $(p, p)$ "; "Multipliers of type $(p, p)$ and multipliers of the group $L_{p}$-algebras"; "Multipliers and the

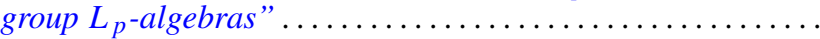

Andrew M. W. Glass, W. Charles (Wilbur) Holland Jr. and Stephen H. McCleary, Correction to: " $a *$-closures to completely distributive lattice-ordered

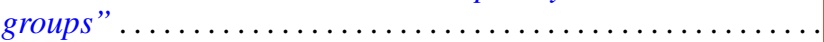

Zvi Arad and George Isaac Glauberman, Correction to: "A characteristic subgroup of

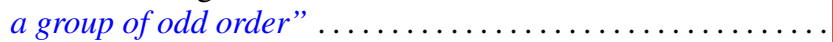

Roger W. Barnard and John Lawson Lewis, Correction to: "Subordination theorems

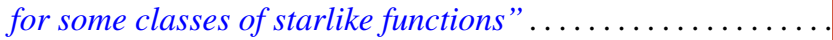

